\title{
Digitized Modeling and Machining Simulation of Equal Base Bevel Gear
}

\author{
Bin Wang ${ }^{1}$, Yunpeng $\mathrm{He}^{1}$, Peiyao Feng ${ }^{1}$, Xun $\mathrm{Sun}^{1}$, Aijun $\mathrm{Xu}^{1}$, Jubo $\mathrm{Li}^{1}$, Tianxing $\mathrm{Li}^{1}$, \\ Xinjie $\mathrm{Jia}^{1}$, and Bo Zhang ${ }^{1}$ \\ ${ }^{1}$ School of Mechatronics Engineering, Henan University of Science and Technology, Luoyang \\ 471003, China
}

\begin{abstract}
In order to achieve the NC machining to the equal base circle bevel gear with a standard cutter, to plan the machining path efficiently and to predict machining interference, based on the equal base circle bevel gear theory, the machining coordinate system for the bevel gear was established, according to gear tooth surface equations of the bevel gears, the tooth surface discretization function was derived, and through the calculation and get the discrete points, the gear surface was modeled in the software UG, and then the planning of the machining path, automatic programming and simulation processing were done. At last, simulation processing, analysis and comparison to the gear surface were carried on with the software VERICUT, improved that the gear surface modeling and $\mathrm{NC}$ machining methods were correct and feasible.
\end{abstract}

\section{Brief introduction of the equal base circle bevel gear}

According to the traditional theory of the equal base circle bevel gear, using the fingershaped milling cutter to machine the bevel gear, through controlling the finger-shaped milling cutter and wheel billet to achieve specific relative motion, to make sure that the base circle radius of the bevel gear at different cone distances is same, the tooth profile will not change suddenly, in which way to machine the entire tooth surface accurately with only one milling cutter. However, since the finger-shaped milling cutter is special tools, the profile accuracy of its shaft cross-sectional shape depends on the accuracy of the sample plate and the technical level of the worker, which means the high production cost and low precision of the cutter, what's more, the cutter must be changed many times due to the wear and tear of the cutters during the machining to large size gear, Because the difficult of making sure the accuracy is consistent to multiple cutters, so, it's more difficult to manufacture the cutter and the manufacturing costs is high, and the gear machining accuracy is low. Therefore, using a common ball end milling cutter to achieve the finishing of the gear surface, which is an effective way to improve the machining accuracy and reduce the manufacturing cost $[1,2]$.

The software UG combines the process of geometric modeling, cutter Location, graphic display and post processing together during the components machining, the problems

Corresponding author: heyunpeng06@163.com 
accrued in programming source, graphic display, cutting simulation and interactive modification of complex components can be effectively solved, which is an effective measure to avoid collision due to the interfere between the machine tool, cutter, and fixture during $\mathrm{CNC}$ machining. Therefore, in order to plan the machining path during the NC programming to the bevel gear and automatically programming in UG software, the digital processing based on the equal base circle bevel gear theory is necessary, and accurate modeling in UG software, this is the key points to the $\mathrm{NC}$ machining to the equal base circle bevel gear with UG software[3,4].

For this reason, based on the equal base circle bevel gear theory, through the establishment of the machining coordinate system, gear surface analysis and discretization, the gear surface modeling was finished in UG software, and further simulation processing to the gear surface was done, in order to verify the correctness of the tooth surface modeling, the CNC machining code generated by UG software was imported into VERICUT software, and simulation processing was done, and the simulation process and the theoretical tooth surface flanks virtual contrast detection was carried on to verified the correctness and feasibility to the tooth surface modeling and NC machining method[5].

\subsection{Characteristics of the equal base circle bevel gear}

By the way of numerical control, the special tooth trace is processed by controlling the finger-shaped milling cutter and wheel billet to achieve specific relative motion, and the bevel gear, whose base circle radius of equivalent gear at different cone distances is same, namely, equal base circle curve bevel gear. The features of these gears are as follows:

$$
r_{v b}=\frac{z m_{t} \cos \alpha_{n}}{2 \cos \delta \cos ^{2} \beta}=\frac{z m_{t e} \cos \alpha_{n}}{2 \cos \delta \cos ^{2} \beta_{e}} \equiv \text { const }
$$

According to the Eq. (1), the relationship between the helix angle $\beta$ of the tooth trace and cone distance $\mathrm{R}$ is:

$$
\beta=\arccos \sqrt{\frac{R \cos ^{2} \beta_{e}}{\mathrm{R}_{\mathrm{e}}}}
$$

where: $z$--the number of teeth for the bevel gear;

$\delta$--the indexing angle of bevel gear $\left(^{\circ}\right)$;

$\alpha_{n}$--the normal pressure angle of tooth profile $\left({ }^{\circ}\right)$;

$\mathrm{R}, \mathrm{Re}-$-cone distance at an arbitrary point and of the big-end respectively $(\mathrm{mm})$;

$m_{t}, m_{t e}$--the transverse modules at the cone distances $\mathrm{R}$ and $\mathrm{Re}(\mathrm{mm})$;

$\beta, \beta_{e}$--the helix angles of tooth trace corresponding to $\operatorname{R}$ and $\operatorname{Re}\left({ }^{\circ}\right)$.

\section{The modeling of equal base circle bevel gears tooth surface}

\subsection{The establishment of machining coordinate system}

Figure 1 shows the relationship between the relative position of the cutter and the blank in the gear cutting coordinate system during the cutting of the equal base circle bevel gears as the ball end milling cutter. While the ball end milling cutter move from the big end to the small end in a certain speed, wheel blanks rotate according in a variable speed regularly, the 
center of the ball end cutter move along line isometric of the actual tooth line, that's how the tooth surface of the equal base circle bevel gears was enveloped.

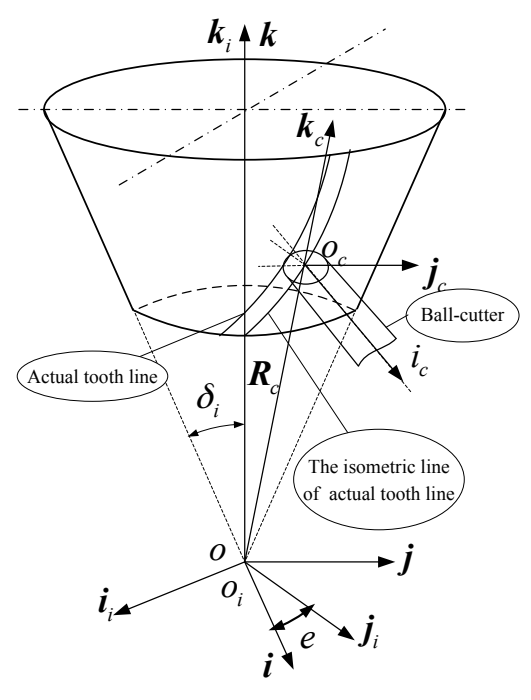

Fig. 1. Gear cutting coordinate system of the equal base circle bevel gears.

$\sigma_{i}\left(O_{i}-i_{i} j_{i} k_{i}\right)$ is the coordinate system fixed to the wheel blanks, the origin $O_{i}$ is at the top of the cone, and the big end of the theoretical tooth line is in the $j_{i} o_{i} k_{i} . \sigma_{c}\left(O_{i}-i_{i} j_{i} k_{i}\right)$ is the coordinate system fixed to the cutter. It is used to describe the cutter surface formed by the rotation of the cutter shaft about axis $i_{c}$. During the cutting process, the tool axis is always perpendicular to the conical busbar, the cutting coordinate system moves along the conical busbar, and $O_{c}$ is located on the conical busbar. $\sigma(O-i j k)$ is the space fixed coordinate system, used to realize the transformation between $\sigma_{i}$ and $\sigma_{c}$, the origin $o$ coincides with the origin $o_{i}$ of the coordinate system of the wheel blanks. During the process of cutting, the tool axis is always in the iok plane of the fixed coordinate system, axis $j_{c}$ and axis $\mathrm{j}$ remain parallel.

The vector relation between the origin $\sigma$ and the origin $\sigma_{c}$ is:

$$
\overrightarrow{O O_{c}}=\overrightarrow{R_{c}}=R_{c}\left(\sin \delta_{i} \vec{i}+\cos \delta_{i} \vec{k}\right)
$$

By sub-cone tooth crown line gear on the rolling relationship between the wheel axis $j_{i}$ coordinate system and the axis $i$ of the fixed axis angle:

$$
e=\theta_{c} / \sin \delta_{i}
$$

In the formulas (3) and (4): $R_{c}$ is the vector between the tool center and the cone tip; $\delta_{i}$ is processed gear root cone angle ( $i=1,2$ corresponding to the small round, big round); $\theta_{c}$ is the polar angle of the tool center at the taper $R_{i}$.

The transformation matrix between each coordinate system is: 


$$
\sigma_{c} \rightarrow \sigma:\left[M_{0 C}\right]=\left[\begin{array}{lll}
\cos \delta_{i} & 0 & \sin \delta_{i} \\
0 & 1 & 0 \\
-\sin \delta_{i} & 0 & \cos \delta_{i}
\end{array}\right], \sigma \rightarrow \sigma_{i}:\left[M_{i 0}\right]=\left[\begin{array}{lll}
\sin e-\cos e & 0 \\
\cos e \sin e & 0 \\
0 & 0 & 1
\end{array}\right]
$$

\subsection{Discretization of tooth flank surface}

The equal base circle bevel gears tooth surface equation was obtained originally based on finger-shaped milling cutter theory. In the cutter coordinate system, the contact point between the tool surface and the gear tooth surface satisfies the meshing equation, and the tooth surface equation of the equivalent equal base circle bevel gear is obtained by solving the meshing equation $[6,7]$ :

$$
\overrightarrow{r^{(i)}}=\left[M_{i O}\right]\left[M_{O C}\right] \overrightarrow{r^{(c)}}+\left[M_{i O}\right] \overrightarrow{R_{c}}
$$

According to the tooth surface equation, substitute the parameters, simplified processing, get the gear tooth surface concave point formula:

$$
\left\{\begin{array}{l}
X=\sin e\left(X_{i}^{(c)} \cos \delta+Z_{i}^{(c)} \sin \delta\right)-Y_{i}^{(c)} \cos e+R_{c} \sin e \sin \delta \\
Y=\cos e\left(X_{i}^{(c)} \cos \delta+Z_{i}^{(c)} \sin \delta\right)+Y_{i}^{(c)} \sin e+R_{c} \cos e \sin \delta \\
Z=-X_{i}^{(c)} \sin \delta+Z_{i}^{(c)} \cos \delta+R_{c} \cos \delta
\end{array}\right.
$$

Where: $R_{c}=\sqrt{R^{2}+\left(r_{0}-s\right)^{2}+2 R\left(r_{0}-s\right)^{2} \sin \beta}$

$X_{i}{ }^{(c)}$ corresponds to $X_{c d}{ }^{(c)}, X_{b c}{ }^{(c)}, X_{a b}{ }^{(c)}$ in $\mathrm{CD}, \mathrm{BC}$ and $\mathrm{AB}$;

$Y_{i}^{(c)}$ corresponds to $Y_{c d}{ }^{(c)}, Y_{b c}{ }^{(c)}, Y_{a b}{ }^{(c)}$ in $\mathrm{CD}, \mathrm{BC}$ and $\mathrm{AB}$;

$Z_{i}^{(c)}$ corresponds to $Z_{c d}{ }^{(c)}, Z_{b c}{ }^{(c)}, Z_{a b}{ }^{(c)}$ in $\mathrm{CD}, \mathrm{BC}$ and $\mathrm{AB}$.

$\mathrm{CD}:\left\{\begin{array}{l}X_{c d}^{(c)}=r k \cdot \cos (T k+s k / r k)-r v \\ Y_{c d}^{(c)}=-r k \cdot \sin (T k+s k / r k) \cdot \cos \gamma \\ Z_{c d}^{(c)}=r k \cdot \sin (T k+s k / r k) \cdot \sin \gamma\end{array}\right.$

$\mathrm{BC}:\left\{\begin{array}{l}X_{b c}^{(c)}=r k \cdot \cos (T k)-r v \\ Y_{b c}^{(c)}=-r k \cdot \sin (T k) \cdot \cos \gamma \\ Z_{b c}^{(c)}=r k \cdot \sin (T k) \cdot \sin \gamma\end{array}\right.$

$\mathrm{AB}:\left\{\begin{array}{l}X_{a b}^{(c)}=r f+R b \cdot[1-\cos (\theta k)]-r v \\ Y_{a b}^{(c)}=-R b \cdot \sin (\theta k) \cdot \cos \gamma \\ Z_{a b}^{(c)}=R b \cdot \sin (\theta k) \cdot \sin \gamma\end{array}\right.$

sk-- the tooth profile modification at the radius of sk;

Rv--radius of the equivalent gear indexing circle $(\mathrm{mm})$;

Tk--The angle between the straight line $O_{a} K$ and the axis of the cutter $\left(^{\circ}\right), O_{a}$ is the equivalent spur gear center ;

$\mathrm{rf}-r_{v}-h_{f}$;

$\mathrm{Rb}-\mathrm{-}$ the radius of the cutter $\operatorname{arc}(\mathrm{mm})$; 
$\theta k$--The center angle between the K point and A point on the arc of the cutter $\left({ }^{\circ}\right)$;

$\boldsymbol{\gamma}$--Tool axis truncation around its axis clockwise rotation angle $\left(^{\circ}\right)$.

\subsection{The establishment of the gear three-dimensional model}

On the base of conical gear tooth surface equation, a series of different values of the cone distance parameter $\mathrm{R}$, according to the above formula of discrete points can get the discrete point coordinates of the tooth surface. Table 1 shows the geometric parameters of the example gear. The calculated instantaneous discrete point coordinates are connected, constituting the tooth surface contact wire. According to this series of contact lines, you can constitute a space curved surface gear. Extracting these discrete points, importing UG can constitute a lamellar tooth surface to complete three-dimensional model of the gear. As shown in figure 2 is the lamellar tooth after finishing.

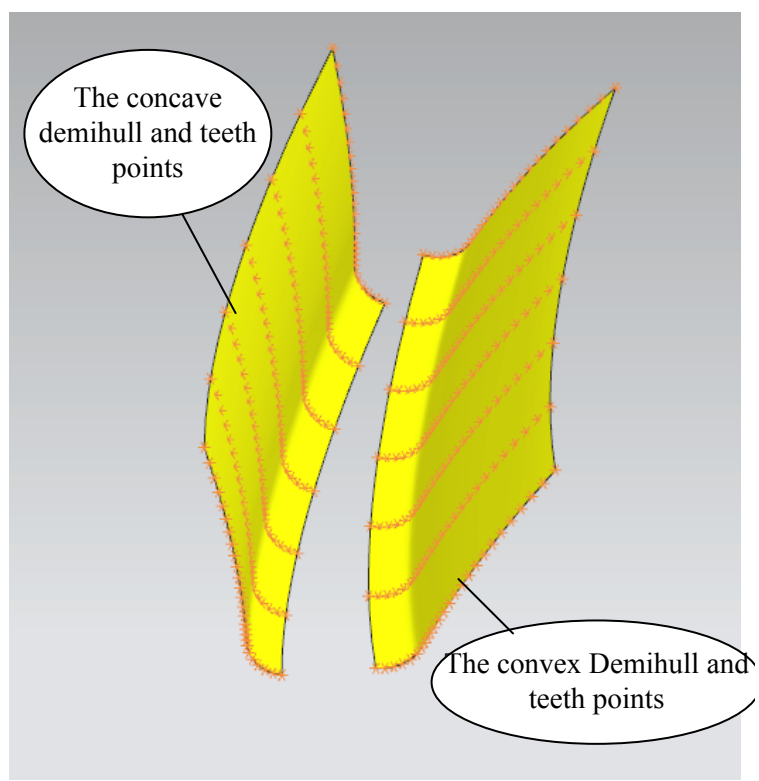

Fig. 2. The lamellar tooth.

Table 1. Basic parameters of gear pair.

\begin{tabular}{|l|l|}
\hline parameters of gear pair & Numerical value \\
\hline Number of pinion $Z_{1}$ & 22 \\
\hline Number of Bull wheel $Z_{2}$ & 27 \\
\hline Big end transverse module $M_{t e}$ & 12 \\
\hline Breadth of tooth B & 40 \\
\hline Big-end helix Angle $\beta$ & $6^{\circ}$ \\
\hline Shaft Angle $\gamma$ & $90^{\circ}$ \\
Normal pressure Angle $\alpha_{n}$ & $20^{\circ}$ \\
\hline
\end{tabular}

As shown in figure 3, generating design gear precise three-dimensional model through the lamellar tooth. 


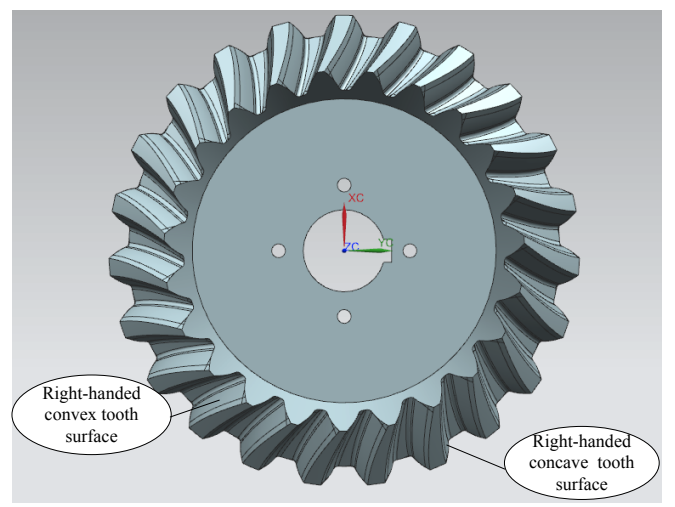

Fig. 3. The design of the three - dimensional model of the equal base circle bevel gears.

\section{Simulation processing}

In order to improve the processing efficiency of the equal base circle bevel gear, the ball milling cutter is only used in the fine finishing, the forming milling which is more efficient is still used in the process of rough machining and semi-finishing. The three-dimensional model of the gear which is semi-finished was built as the gear blank which was then imported to the software UG.

The coordinate system for fine finishing was set in the gear blank. The ball milling cutter was chosen and built as the finishing tools, the parameters of the cutter was set and the diameter of the cutter is $6 \mathrm{~mm}$. Select [Cavity milling] as the processing methods for the finishing machining, select [Fixed shaft contour milling] in the subtypes. Select the convex surface of the gear as the cutting area. Set the driving method as [streamline driver], use the streamline driving method to make sure the cutter move in the radial direction along the cutting routes during the machining processing, in which way the finishing process to the gear surface can be done, select reciprocating cutting as the cutting mode for the finishing machining, set the step distance as the residual height, then generate the tool path for the finishing machining to gear convex surface, set the remaining machining parameters, then do the simulation processing (figure 4), verify the correctness of the gear processing method initially. Generated NC codes suit for the cutting machine through the postprocessing, and put the NC codes above into the software VERICUT, in which further simulation and validation can be done. The simulation results were shown in figure 5[8].

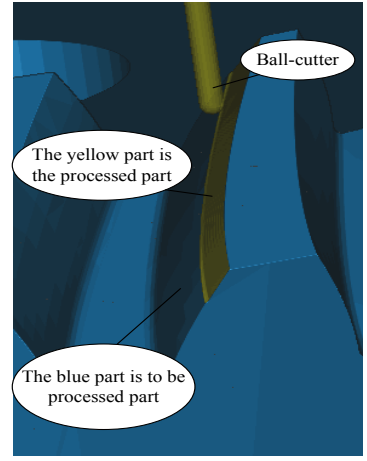

Fig. 4. UG software simulation processing view. 


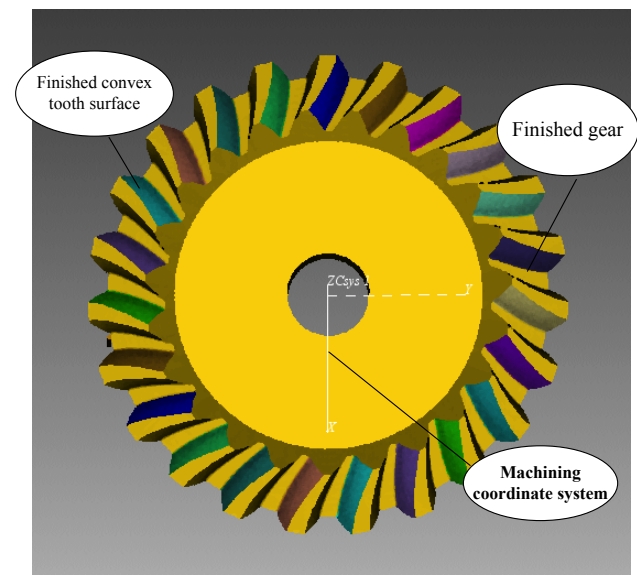

Fig. 5. VERICUT software simulation of the processed gear.

The simulated tooth surface and the theoretical tooth surface were compared and analyzed, Figure 6 shows the result. In the figure, the red area is overcut, the blue area is undercut, and the error less than $0.01 \mathrm{~mm}$ is displayed in green. As can be seen from figure 6 , there is a slight overcut of the concave end of the gear tooth and a small amount of undercut at the large end. A small amount of overcut is machined on the large end of the convex end, and the rest is overlapped with the theoretical tooth surface. Figure 6 shows a slight error between the machining flank and the theoretical flank, which can be improved by controlling the tool pitch and tool diameter. The analysis and comparison results show that the method of tooth surface modeling of the equal base circle bevel gear and its processing method are correct and feasible[9].

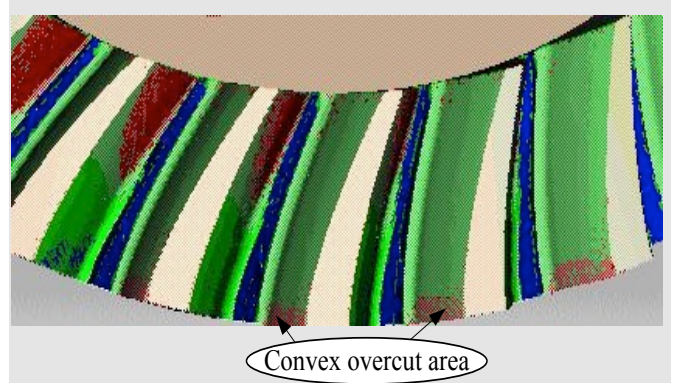

Fig. 6. Tooth surface precision analysis after processing.

\section{Conclusion}

According to the theory of the equal base circle bevel gear, the tooth surface of the equal base circle bevel gear is completed in UG software through discretization of the surface of the equal base circle bevel gear, and is simulated in UG. Finally, VERICUT software is used for further simulation of CNC machining and verification, the results show that:

1).Based on the tooth surface equation, the calculation, extraction, introduction and $3 \mathrm{D}$ modeling of the equivalent base tooth surface are correct.

2).Based on the ball-end milling cutter, the NC machining of the equal base circle bevel gears in the UG software is automatically programmed, the $\mathrm{NC}$ code and the simulation machining are generated correctly and practically. 
Acknowledgement: The research reported here is partially supported by the National Natural Science Foundation of China (Grant No.51405135,51575161,U1504522), Henan Universities Key Scientific Research Project of China (Grant No.15 A460003,17A460002,16A460033),The Doctoral Scientific Research Foundation of Henan University of Science and Technology.

\section{Reference}

1. Gong Y P. Study on Transmission Theory, Strength Analysis and Running-in Simulation of Equal Base Circle Bevel Gear [D]. Shenyang: Northeastern University 1996.

2. Cai Chunyuan, Cheng Naishi. Principle of Equal Base Circle Bevel Gear [J]. Chinese Journal of Construction Machinery, 1992, 28 (3): 61-64.

3. Zhang W H, Gong Y P, Wang D. Analysis of Tooth Geometry and Modification of Equal Base Circle Bevel Gear [J]. Journal of Northeastern University (Nature Science) 2000, 21(4): 390-393.

4. Wang B, Deng X Z, Dang Y G. Processing Principle and Tooth Surface Determination of Equal Base Circle Bevel Gear Using Disc Cutter, Journal of Aerospace Power, 2014, 29(3): 663-671.

5. Wang B, Deng X Z, Jia X J. Calculation of Tool State and Trajectory Processing Equal Base Circle Bevel Gear Using Disc Cutter [J]. China Mechanical Engineering, 2013, 24(19): 2664-2670.

6. Wang Fulin, Yi Chuanyun, Wang Tao. et a1. A generating method for digital gear tooth surfaces[J]. Int Adv Manuf Technol (2006) 28: 474-485.

7. Wang Bin, Fan Mingxing, Sun Xun. Cutter position calculation of machining equal base circle bevel gears with a pot-shaped milling cutter[J]. International Journal of Advance Manufacturing Technology, 2016: 87, 2625-2637.

8. Kong Deying, Chong Kai, Wang Xiao. NC machining simulation and optimization based on UG / CAM and VERICUT [J]. Machine Tool \& Hydraulics,2011,39 (23): 147-149+153.

9. Wang Bin, Deng Xiaozhong, Chen Jun. Simulation for the NC machining to the equal base circle bevel gear with figer-shaped cutter based on VERICUT software [J]. Manufacturing technology and machine tools, 2013, (09): 124-128. 